\title{
Quelle place pour les petits exploitants dans l'agriculture contractuelle? Le cas de la tomate industrielle en Algérie
}

\author{
Sami Assassi ${ }^{1, *}$, Ali Daoudi ${ }^{1}$ et Jean-Philippe Colin ${ }^{2}$ \\ ${ }^{1}$ École nationale supérieure agronomique (ENSA), Alger, Algérie \\ 2 Institut de recherche pour le développement (IRD), UMR GRED, Montpellier, France
}

\begin{abstract}
Résumé - Les travaux portant sur l'inclusion des petits exploitants dans l'agriculture contractuelle dans le monde dressent des constats contradictoires. En Algérie, l'État a engagé en 2009 un programme d'appui à l'intégration des filières lait et tomate industrielle, en accordant des primes aux entreprises et aux agriculteurs qui s'engagent ensemble dans des contrats de commercialisation. L'adhésion des agriculteurs et des entreprises à ce dispositif contractuel a été importante dès son lancement, mais qu'en est-il du développement de contrats de production et de l'intégration des petits producteurs? Nous avons étudié le dispositif contractuel mis en place par la plus grande conserverie de tomate en Algérie (CAB) dans le plus grand bassin de production de tomate industrielle du pays (wilaya de Guelma), avec des enquêtes portant sur la moitié de ses contractants (184/368) et des producteurs hors contrat de la région (58/115). L'étude témoigne du rôle déterminant de la prime dans l'adhésion de l'entreprise et des agriculteurs au contrat de commercialisation. Elle augmente le prix aux producteurs et les motive à livrer leur production à l'entreprise, sécurisant ainsi les approvisionnements de cette dernière. Cette forte participation au contrat de commercialisation a incité la $\mathrm{CAB}$ à proposer aux agriculteurs des contrats de production. Les petits producteurs, pourtant majoritaires, y participent très peu, pour des raisons mises en évidence dans l'article.
\end{abstract}

Mots clés : agriculture contractuelle / agro-industrie / inclusion / petits producteurs / tomate industrielle

Abstract - What place for smallholders in contract farming? The case of the canned tomato in Algeria. The studies tackling the question of the inclusion of smallholders in contract farming around the world raise contradictory observations. In Algeria, the State has launched since 2009 a program to support the integration of the milk and canned tomato sectors by granting premiums to firms and farmers who enter into a marketing contract. Firms and farmers' involvement in contract farming was significant from the start, but what about the development of production contracts and the inclusion of smallholders? We studied the contractual arrangement proposed by the largest Algerian tomato cannery (CAB) in the largest industrial tomato production area in the country (wilaya of Guelma) and surveyed half of its contractors (184/368) and of non-contract producers in the region (58/115). The study highlights the key role of the premium in firms and farmers' participation in the marketing contract. It improves the price for producers and motivates them to deliver their production to the company, thereby securing the latter's supplies. This strong participation in the marketing contract prompted the CAB to offer farmers production contracts. However, smallholders, even though by far the most numerous tomato producers, are marginally involved in those contracts, for reasons highlighted in the article.

Keywords: contract farming / agro-industry / inclusion / smallholders / canned tomato

\section{Introduction}

L'agriculture contractuelle (AC) est l'un des dispositifs à travers lesquels les entreprises agroalimentaires intègrent dans

\footnotetext{
*Auteur de correspondance : assassisami@hotmail.com
}

leurs chaînes de valeur des millions de producteurs agricoles, avec une dynamique renforcée ces dernières décennies dans les pays en développement (Barrett et al., 2012). L'agriculture contractuelle est entendue au sens d'un accord, écrit ou oral, entre un agriculteur et une entreprise, prévoyant une ou plusieurs conditions de commercialisation, voire de production, d'un produit agricole (Roy, 1963). 
L'intérêt des chercheurs pour l'agriculture contractuelle ne cesse de grandir, avec des perspectives d'analyse qui évoluent. Les évaluations "globalisantes» réalisées au niveau national, dominantes entre les années 1970 et 1990, cèdent de plus en plus la place, à partir des années 2000 , à des études ciblées sur les effets de l'agriculture contractuelle sur les participants (Prowse, 2012). Trois grandes thématiques en particulier sont récurrentes dans les travaux récents:

- l'évaluation des effets de l'agriculture contractuelle sur les agriculteurs impliqués. Des effets positifs sont globalement relevés sur les revenus des ménages, leur sécurité alimentaire, l'intégration au marché et le changement technique (Burnod et Colin, 2012; Soullier et Moustier, 2018);

- les conditions d'exécution des contrats et l'efficacité organisationnelle de l'agriculture contractuelle. Les résultats sont ici moins convergents, entre la réduction des coûts de transaction (Bellemare et Bloem, 2018) et des risques de comportements opportunistes des entreprises ou des agriculteurs dans certaines situations (Ton et al., 2018);

- l'inclusion des petits producteurs dans l'agriculture contractuelle. Là aussi les constats sont contradictoires, entre inclusion effective des petits producteurs (Barrett et al., 2012) et exclusion (Ito et al., 2012).

Cet article traite de ce dernier point: la question de l'inclusion des petits producteurs dans l'agriculture contractuelle, question centrale dans les débats sur la restructuration des filières agroalimentaires dans les pays en développement (Burnod et Colin, 2012).

Dans un numéro spécial de World Development, Reardon et al. (2009) proposent une grille d'analyse, actualisée ensuite par Barrett et al. (2012), qui traite de cette question en deux temps : les déterminants de l'engagement, ou pas, des acteurs dans l'agriculture contractuelle, et l'inclusion, ou pas, des petits producteurs dans l'agriculture contractuelle.

Pour Reardon et al., l'engagement des acteurs dans l'agriculture contractuelle dépend de deux principales considérations : leurs capacités à réaliser les investissements que l'agriculture contractuelle exige et les avantages et les inconvénients de l'agriculture contractuelle-les acteurs y participent lorsque leur perception de l'utilité attendue de cette adhésion dépasse celle du fait de rester hors contrat (Barrett et al., 2012). À cet égard, les avantages de l'agriculture contractuelle pour les productions périssables, qui requièrent une coordination étroite pour la récolte, la livraison et la sécurisation des débouchés, sont souvent notés (Minot et Sawyer, 2016). L'inclusion des petits producteurs dans l'agriculture contractuelle est vue par ces auteurs comme dépendant essentiellement de la configuration de la structure agraire, entre petits et grands producteurs. Barrett et al. (2012) notent que si elles ont le choix, les entreprises contractent avec les agriculteurs leur permettant de réduire les prix d'achat de la matière première et les coûts de transaction. Même si les constats sont contradictoires relativement aux prix, il est admis que les coûts de transaction sont plus élevés lorsque les entreprises contractent avec un grand nombre de petits agriculteurs. Reardon et al. (2009) relèvent des exceptions à cette tendance, qu'ils résument en quatre points :
- ayant moins d'options de vente, les petits producteurs sont une source d'approvisionnement moins risquée pour l'entreprise ;

- ces producteurs sont plus aptes à s'investir dans les productions à haute intensité de main-d'œuvre ;

- les coûts de transaction pour l'entreprise peuvent être réduits si les petits producteurs s'organisent en coopératives de commercialisation;

- leur problème d'accès au crédit peut être surmonté si les entreprises proposent des contrats de production.

La participation des petits exploitants peut être aussi facilitée par l'État, lorsque ce dernier facilite leur accès aux facteurs de production (Burnod et Colin, 2012), et par la répétition des transactions, qui crée une proximité relationnelle avec les entreprises et réduit les incertitudes, et, par conséquent, les coût de transaction (Moustier, 2012).

En mobilisant cette grille d'analyse, nous questionnons dans cet article le caractère inclusif, ou non, du dispositif contractuel mis en place par la plus grande conserverie de tomate en Algérie en termes de part de marché et capacité de transformation, la Conserverie Amor Benamor (CAB), dans le premier bassin en termes de production de tomate industrielle (TI), la wilaya de Guelma. Cette question fait tout particulièrement sens dans le contexte algérien, au vu de la structure productive nationale dominée par les petits exploitants : plus de $50 \%$ des agriculteurs du pays exploitent moins de 5 ha, et plus de $70 \%$, moins de 10 ha (Ministère de l'Agriculture et du Développement rural, 2003). La question fait sens également car l'agriculture contractuelle, introduite récemment, est en expansion sous l'effet d'une politique publique volontariste axée sur l'offre et la subvention d'un contrat de commercialisation (CC) facilement accessible à tous.

Le bilan de cette politique est positif. En 2014, toutes les conserveries algériennes (19) ont adhéré au programme. Le nombre d'agriculteurs participant à ce dispositif est en augmentation continue. En 2014, près de $80 \%$ des superficies cultivées en tomate industrielle à l'échelle nationale (15 $600 \mathrm{de}$ 19700 ha) étaient sous contrat ( $87 \%$ à Guelma); plus de $76 \%$ des adhérents étaient des petits producteurs $(\leq 5 \mathrm{ha})$.

En plus de documenter cette adhésion massive au contrat de commercialisation, cet article décrypte la démarche globale de contractualisation de la $\mathrm{CAB}$, évalue la place réservée aux petits producteurs et analyse les déterminant de leur participation au dispositif contractuel.

\section{Matériels et méthodes}

\subsection{Contexte de l'étude : libéralisation de la filière, crise de coordination et choix de l'agriculture contractuelle}

Les réformes libérales des années 1980-1990 ont profondément restructuré la filière de la tomate industrielle en Algérie, avec l'éclatement des grands domaines agricoles publics en petites exploitations privées (Baci, 1999), la libéralisation du commerce agricole (Assassi et al., 2017) et la concentration des conserveries selon le processus de «Ucurve» décrit par Reardon et al. (2009) (prééminence de 


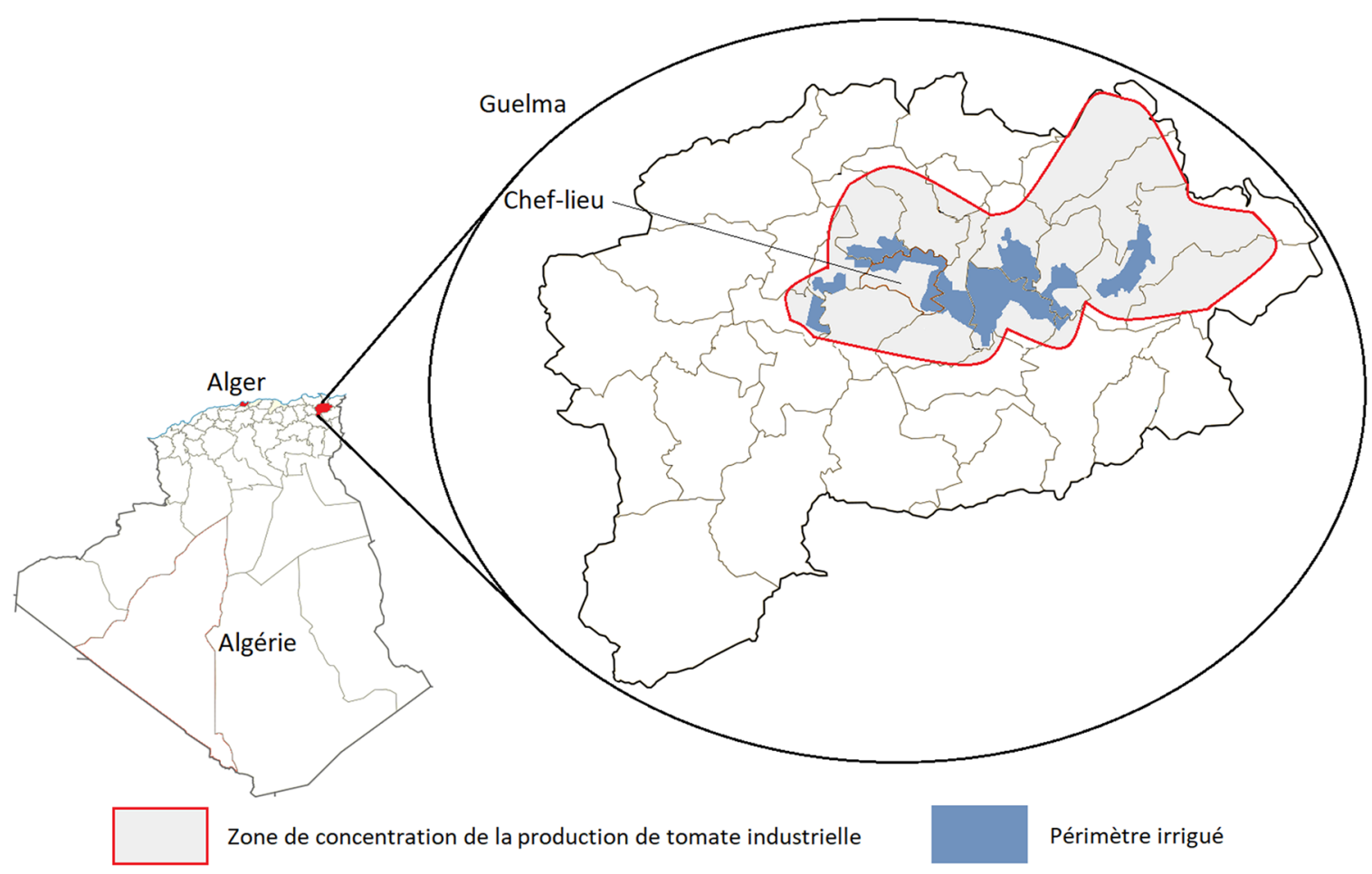

Fig. 1. Localisation de la région d'étude et de la zone de production de tomate industrielle. Source : auteurs, à partir des données de la Direction des services agricoles (2014).

Fig. 1. Location of the study region and the canned tomato production area.

grandes entreprises publiques, puis émergence de nombreuses petites firmes privées, et ensuite concentration en grandes entreprises privées). Ces réformes ont fortement perturbé la filière de la tomate industrielle entre 2000 et 2009, avec une production décroissante $(-3 \%$ en moyenne annuelle) et variable (coefficient de variation de $20 \%$-ministère de l'Agriculture et du Développement rural, 2000 à 2009). Le recours des conserveries à l'importation de triple concentré de tomate, pour la fabrication du double concentré, s'est intensifié. Ce produit représentait près de $49 \%$ des approvisionnements de la CAB en 2014.

Pour relancer la filière, l'État a mis en place en 2009 un dispositif contractuel hybride public-privé (Burnod et Colin, 2012), sous forme de contrat de commercialisation engageant les agriculteurs à livrer et les entreprises à recevoir la quantité qu'ils décident de mentionner dans le contrat, avec un prix fixe, indépendant de la qualité. Pour les inciter à coordonner leurs transactions dans ce cadre, l'État offre aux agriculteurs et aux entreprises des primes d'intégration de respectivement 4 et $1,5 \mathrm{DZD} / \mathrm{kg} \quad\left(1 €=145 \mathrm{DZD}\right.$, taux de change du $1^{\mathrm{er}}$ juillet 2020 ), couvrant ainsi $37 \%$ du prix au producteur $(15 \mathrm{DZD} / \mathrm{kg}$, resté inchangé depuis 2014). Depuis cette date, un simple procès-verbal de constat de l'installation de la culture, établi par les agents de la Direction des services agricoles de la wilaya, suffit aux agriculteurs pour signer un contrat avec une conserverie et bénéficier de la prime.

L'introduction de l'agriculture contractuelle dans la filière a restructuré et réduit considérablement le marché au comptant de la tomate industrielle, c'est-à-dire toutes transactions sans engagement préalable et basées sur des prix spot, marché désormais animé par un nombre réduit de collecteurs privés.
Ces derniers achètent aux agriculteurs, avec des transactions spot, pour le compte des conserveries éloignées des bassins de production, qui s'engagent à réceptionner les quantités livrées et à leur assurer une commission fixe. Les prix que proposent ces collecteurs sont moins élevés ( $13 \mathrm{DZD} / \mathrm{kg}$ en général) que ceux pratiqués dans le cadre de l'agriculture contractuelle (15 DZD/kg en 2019).

\subsection{La wilaya de Guelma : bassin de production de tomate industrielle}

Situé au nord-est du pays, le site d'étude de Guelma représente en 2014, au plan national, $19 \%$ des superficies de tomate industrielle (3752 ha pour 700 producteurs), $25 \%$ de la production $(262000 \mathrm{t}), 19 \%$ des contractants, $21 \%$ des superficies sous contrat et $30 \%$ des capacités de transformation (316 $000 \mathrm{t} /$ saison de récolte) (ministère de l'Agriculture, 2014). La CAB, qui détient $93 \%$ de ces capacités de transformation, possède une unité à Skikda et deux à Guelma, dont la capacité de transformation dépasse largement la production totale de la wilaya. Ces surcapacités sont le résultat d'une opportunité de financement que la CAB a saisi : crédits bonifiés et exonérations fiscales sur les équipements importés. La production locale de tomate industrielle est disputée par les autres conserveries, dont trois situées à Guelma. La culture de la tomate industrielle est concentrée sur un périmètre irrigué aménagé par l'État; elle est souvent conduite en rotation avec le blé ou la pomme de terre (Fig. 1). Les producteurs sont majoritairement des locataires informels. 


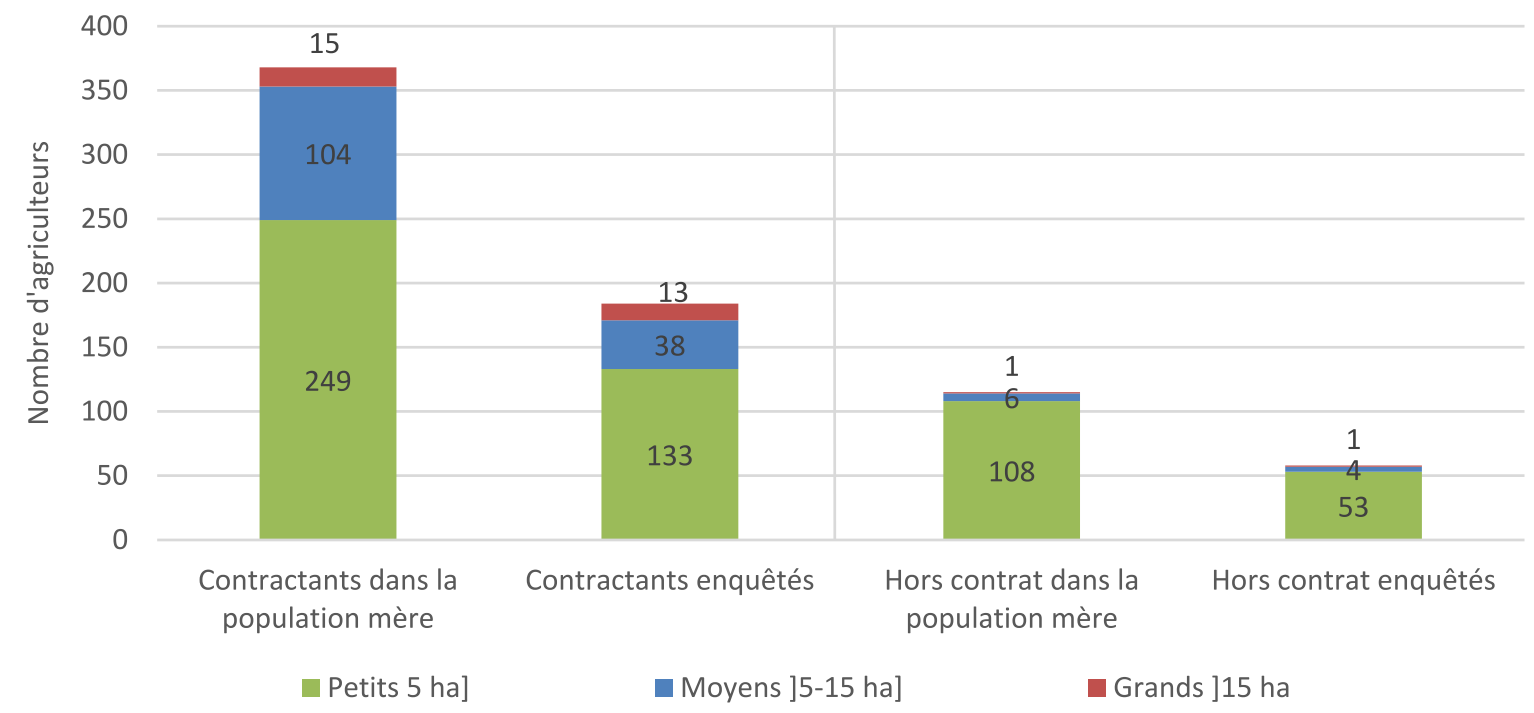

Fig. 2. Échantillon des producteurs interrogés.

Fig. 2. Sample of producers surveyed.

\subsection{Méthodologie, données et enquêtes}

Le caractère inclusif ou non du dispositif contractuel de la $\mathrm{CAB}$ a été apprécié en deux temps : présentation du dispositif (contrats et conditions d'accès) et des choix des acteurs en premier lieu, puis analyse des déterminants de ces choix. Les relations de causalité entre les choix des contrats et leurs déterminants ont été établies grâce aux analyses descriptives basées sur les déclarations des acteurs relatives aux raisons de participer, ou non, à l'agriculture contractuelle. Les résultats obtenus ont été ensuite validés via l'analyse des pratiques effectives et leurs déterminants : comparaisons des différents modes de coordination en termes de prix, qualité et disponibilité de la matière première, risque et délais de livraison et de paiement. Pour une meilleure compréhension des pratiques des agriculteurs, une typologie a été réalisée à l'aide d'une Analyse des correspondances multiples (ACM), une méthode factorielle permettant d'examiner les relations entre des variables catégorielles (Le Roux et Rouanet, 2010).

Les données utilisées ont été recueillies auprès des structures, centrales et déconcentrées, des ministères de l'Agriculture, ou collectées directement auprès des agriculteurs et de l'entreprise. Les bases de données de la Direction des services agricoles (liste exhaustive des producteurs de tomate industrielle à Guelma entre 2012 et 2014 et leur superficie) et de l'Office national interprofessionnel des légumes et viandes (conserveries contractantes, localisation et quantité livrée par tous les producteurs de tomate industrielle sous contrat en 2014 à l'échelle nationale) ont été utilisées pour une première analyse et la préparation de l'enquête auprès des producteurs. Plusieurs entretiens avec le Directeur général et le responsable du service approvisionnement de la $\mathrm{CAB}$ (portant sur les déterminants du choix de l'agriculture contractuelle, des types de contrats et des types d'agriculteurs) et une enquête par questionnaire ont été réalisés entre 2015 et 2016 par le premier auteur de ce texte.

L'enquête auprès des producteurs a porté sur un échantillon de 184 producteurs parmi les 368 contractants de la $\mathrm{CAB}$ à Guelma en 2014, et de 58 des 115 agriculteurs restés hors dispositif contractuel que comptait la wilaya en 2014. Ces derniers vendent leur production aux entreprises à travers d'autres contractants, ou aux collecteurs privés via des transactions spot. Vu la grande hétérogénéité en termes de superficie cultivée en tomate industrielle pour les deux populations, plusieurs strates de superficie basées sur les normes locales ont préalablement été définies. Un échantillon proportionnel aléatoire a ensuite été tiré dans chacune d'elles (Fig. 2).

Le questionnaire comptait plus de 150 variables temporelles couvrant la période entre 2009 et 2015 , portant sur:

- la structure de l'exploitation (superficie, activités, ménage);

- les performances (production, coût, bénéfice);

- la logique de commercialisation;

- les rapports contractuels avec les entreprises (historique de participation, type de contrat, quantités livrées).

\section{Résultats}

\subsection{Le dispositif contractuel de la CAB et l'adhésion des petits producteurs}

$\mathrm{La} \mathrm{CAB}$, qui vise à augmenter sa production de double concentré de tomate (produit fini), combine l'approvisionnement en tomate industrielle locale et l'importation de triple concentré. Les dirigeants de l'entreprise affirment cependant qu'ils ont fait le choix de privilégier la production locale. Le recours à l'agriculture contractuelle, plutôt qu'au marché au comptant, est le moyen de pérenniser ce choix. Au même titre que les autres conserveries, la CAB propose depuis 2009 le contrat de commercialisation de l'État à tous ses fournisseurs qui répondent aux conditions d'éligibilité à la prime. En plus des services de ce contrat (engagement d'achat, prix fixe, prime), la $\mathrm{CAB}$ propose à des agriculteurs sélectionnés parmi les adhérents au contrat de commercialisation des services d'appui à la production, une démarche qu'elle avait engagée à petite échelle dès 2003 (15 à 20 producteurs, selon les années). 
Tableau 1. Distribution des contrats par classe de superficie.

Table 1. Distribution of contracts by area class.

\begin{tabular}{lllll}
\hline & Petits producteurs & Moyens producteurs & Grands producteurs & Effectif total \\
\hline CC & $80 \%$ & $54 \%$ & $43 \%$ & 133 \\
CPA-A & $20 \%$ & $38 \%$ & $36 \%$ & 45 \\
CPA-B & $0 \%$ & $8 \%$ & $21 \%$ & 6 \\
Effectif total & 133 & 37 & 14 & 184 \\
\hline
\end{tabular}

En fonction de la gamme de services proposés, deux types de contrats de "production assistée» peuvent être distingués (en reprenant la terminologie de Burnod et Colin, 2012). Dans le premier contrat de production assistée (CPA-A), la CAB fournit des plants en motte à crédit à hauteur de $50 \%$ de leur valeur, accorde des avances en espèces pour l'achat de quatre quintaux d'engrais par hectare (soit $50 \%$ des besoins de fertilisation), loue à crédit des récolteuses mécaniques et assure aussi du conseil technique à la demande; tous les crédits sont attribués sans intérêt. Pour accéder à ce contrat, en plus des conditions administratives requises pour le contrat de commercialisation, les agriculteurs doivent justifier d'une ancienneté d'au moins quatre ans de collaboration avec l'entreprise, au cours desquels ils doivent avoir livré au minimum $70 \%$ de leur production annuelle (estimée sur la base d'un rendement moyen de référence de $400 \mathrm{q} / \mathrm{ha}$ ). En 2014, 44 producteurs ont reçu des plants en motte à crédit et un prêt en espèces, deux d'entre eux bénéficiant en sus de conseils techniques, non demandés par les autres producteurs, et trois autres ont loué des récolteuses de la CAB.

Dans le deuxième type de contrat de production assistée (CPA-B), les plants en motte sont fournis entièrement à crédit, l'avance en espèces équivaut à la valeur des huit quintaux d'engrais requis par hectare. La CAB assure aussi un appui technique personnalisé à la demande (suivi, essais, formation), loue à crédit des récolteuses et vend à crédit, sur plusieurs années et sans intérêt, des planteuses et des récolteuses. Pour accéder à ce contrat, les agriculteurs doivent justifier, en plus des conditions du contrat de commercialisation, d'au moins dix ans de collaboration ininterrompue avec l'entreprise, au cours desquels ils doivent avoir livré la totalité de leur production à la CAB. La superficie en tomate industrielle cultivée par les producteurs souhaitant accéder aux crédits d'achat d'une récolteuse doit être supérieure à 20 ha, pour des raisons de rentabilité de l'investissement et donc de capacité de remboursement (le prix d'une récolteuse est de 12 millions de DZD, soit plus de $82000 €$ ). En 2014, six producteurs ont bénéficié de plants en motte à crédit, deux d'entre eux ont reçu aussi un prêt et deux autres un appui technique. Aucun producteur n'a acheté de machine agricole en 2014 ; avant cette date, cinq récolteuses et 24 planteuses ont été vendues dans le cadre de ce contrat.

Le contrat de commercialisation domine largement dans toutes les catégories de producteurs, notamment les petits (Tab. 1). Le CPA-A concerne $24 \%$ des agriculteurs interrogés, dont $58 \%$ sont des petits producteurs. Le CPA-B, dont les services sont adaptés aux grandes surfaces, n'a concerné qu'un nombre très réduit de moyens et grands producteurs depuis son lancement. Restant marginal et inaccessible aux petits producteurs, il ne sera pas analysé.
La participation aux différents contrats n'est pas systématique: certains agriculteurs sont restés hors contrat de commercialisation, les contrats de production ne sont pas ouverts à tous et certains agriculteurs éligibles aux CPA-A sont restés sous contrat de commercialisation. De ce fait, l'analyse du caractère inclusif, ou pas, du dispositif contractuel de la $\mathrm{CAB}$ nécessite l'examen, au-delà du nombre de petits producteurs dans les différents types de contrats, des déterminants de leur adhésion aux contrats de commercialisation et CPA-A.

\subsection{Logiques des acteurs en présence}

\subsubsection{Perspectives de l'industriel}

Le choix de l'approvisionnement en tomate industrielle locale plutôt qu'en triple concentré vient de deux considérations :

- le prix de la tomate industrielle locale, stable et moins élevé (notamment grâce à la prime) comparé à celui du triple concentré, variable et tendant à augmenter (Tab. 2);

- la qualité relativement meilleure du produit fini que permet d'obtenir la tomate fraîche.

La production de tomate industrielle locale étant insuffisante, la $\mathrm{CAB}$ recourt par défaut à l'importation de triple concentré pour compléter son approvisionnement.

Relativement à l'option d'un approvisionnement local en tomate industrielle, le recours à l'agriculture contractuelle s'explique par le risque élevé que présente le marché au comptant. Les quantités sur ce dernier sont faibles (concurrence et préférence des agriculteurs pour le contrat de commercialisation) et variables (pas d'engagement), et les prix sont plus élevés et relativement volatils. Grâce à la prime, qui couvre 5,5 des $15 \mathrm{DZD} / \mathrm{kg}$ payés aux agriculteurs, le prix que débourse réellement l'entreprise dans le cadre du contrat $(9,5 \mathrm{DZD} / \mathrm{kg})$ est plus faible que celui pratiqué sur le marché au comptant (13 DZD/kg en moyenne-Tab. 2).

Les choix des types de contrat et des agriculteurs à impliquer dans le dispositif contractuel sont déterminés par la prime et la structure productive locale. L'adoption à grande échelle du contrat de commercialisation subventionné et son accessibilité à tous vient de l'objectif de la $\mathrm{CAB}$ d'augmenter son approvisionnement en tomate industrielle et sa propre part de subvention, de la présence dominante des petits producteurs et du coût très faible du contrat de commercialisation. Ce dernier point est à mettre en rapport avec l'absence d'appui à la production par l'entreprise et les faibles coûts de transactionles agriculteurs se présentent eux-mêmes à l'entreprise pour signer les contrats, dont l'exécution est garantie par le 
Tableau 2. Prix d'achat de la matière première selon les modes d'approvisionnement.

Table 2. Purchase prices of raw material according to the modes of supply.

\begin{tabular}{lccc}
\hline & \multicolumn{2}{c}{ Estimation du prix de revient d'un kilo de produit fini (DZD) } \\
\cline { 2 - 4 } & $\begin{array}{l}\text { Tomate fraîche } \\
\text { locale avec contrat } \\
\text { et subvention }\end{array}$ & $\begin{array}{l}\text { Tomate fraîche sur le } \\
\text { marché au comptant }\end{array}$ & $\begin{array}{c}\text { Prix du TCT }^{* *} \text { importé } \\
\text { arrivée usine }\end{array}$ \\
\hline 2008 , avant l'AC & $/$ & 50 & 61 \\
2014 & 47,5 & 65 & 89 \\
\hline
\end{tabular}

${ }_{* *}^{*} 1 \mathrm{~kg}$ de produit fini $=5 \mathrm{~kg}$ de tomate fraîche $=0,7 \mathrm{~kg}$ de triple concentré de tomate.

${ }^{* *}$ Triple concentré de tomate.

Tableau 3. Avantages et inconvénients du dispositif contractuel et du marché au comptant.

Table 3. Advantages and disadvantages of the contractual arrangement and the spot market.

\begin{tabular}{|c|c|c|c|}
\hline & Marché au comptant & $\mathrm{CC}$ & CPA-A \\
\hline Prix de vente & $\begin{array}{l}\text { Variations intra- et interannuelles. } \\
\text { Prix moyen en } 2014: 13 \text { DZD (10 à } 14 \text { DZD) }\end{array}$ & & Prix fixe: $15 \mathrm{DZD} / \mathrm{kg}$ \\
\hline Débouchés & Risque élevé pour les grands volumes & & Aucune limitation de volume \\
\hline Délais de paiement & Aucun & & $\begin{array}{l}50 \%(7,5 \mathrm{DZD} / \mathrm{kg}) \text { à la livraison } \\
50 \%(7,5 \mathrm{DZD} / \mathrm{kg}) \text { payés } 3 \text { mois après }\end{array}$ \\
\hline
\end{tabular}

paiement ex post (après livraison) de la prime. La généralisation de ce contrat de commercialisation a cependant nécessité, pour la $\mathrm{CAB}$, des investissements en capacités supplémentaires de réception de tomate fraîche (tapis de réception).

La proposition de contrats de production différenciés, dont l'accès est sélectif, tente quant à elle d'attirer et de fidéliser les producteurs jugés professionnels. Ces contrats ont cependant un coût plus important pour la CAB. Pour accompagner les contractants, l'entreprise a créé une filiale en charge du développement agricole, dotée d'une grande pépinière moderne ( 40 millions de plants en motte de variétés sélectionnées par an en 2014), d'un parc de matériel agricole (planteuses, récolteuses) pour la location et d'un staff technique assurant le conseil agricole. L'entreprise mobilise aussi d'importantes sommes d'argent (101 millions de DZD en 2014) pour préfinancer l'acquisition des intrants par les adhérents à ces contrats.

Le coût des contrats de production explique le choix de la $\mathrm{CAB}$ de les réserver aux producteurs solvables. Aucune barrière structurelle, notamment de taille d'exploitation, n'est en revanche évoquée pour le CPA-A ; il peut bénéficier à tous les agriculteurs, y compris les petits. Ce choix inclusif s'explique par deux facteurs importants: la présence dominante des petits producteurs à Guelma et l'existence de garanties d'exécution de ce contrat. La généralisation du contrat de commercialisation à partir de 2009 a créé en effet les conditions d'exécution de ce type de contrat sans coût rédhibitoire pour l'entreprise :
- l'atrophie du marché au comptant limite considérablement les ventes hors contrat;

- la CAB s'est imposée comme le principal acheteur de tomate industrielle à Guelma;

- la pratique du contrat de commercialisation a permis de mieux connaître les agriculteurs et de repérer les plus solvables ;

- le paiement ex post de la prime par l'entreprise prévient tout risque d'aléa moral-les crédits accordés aux producteurs sont inférieurs à la valeur globale de la prime qu'ils perdraient en cas de non-respect du contrat. La valeur moyenne des subventions encaissées par les contractants de la $\mathrm{CAB}$ est trois fois plus importante que les crédits attribués dans le CPA-A et 1,5 fois plus importante que ceux attribués dans le cadre du CPA-B.

\subsubsection{Perspectives des agriculteurs}

Les avantages du contrat de commercialisation et CPA-A sont clairement supérieurs à ceux du marché au comptant (Tab. 3). Le prix est plus élevé et fixe, les débouchés pour la récolte - périssable - sont garantis et le CPA-A offre l'accès à un appui à la production (crédits, conseil). La seule limite de ces contrats concerne le délai de paiement, qui peut atteindre trois mois du fait des conditions de versement de la prime par l'État. Pour réduire ce problème, la $\mathrm{CAB}$ assure le paiement immédiat de $50 \%$ de la valeur de la marchandise livrée, pour 


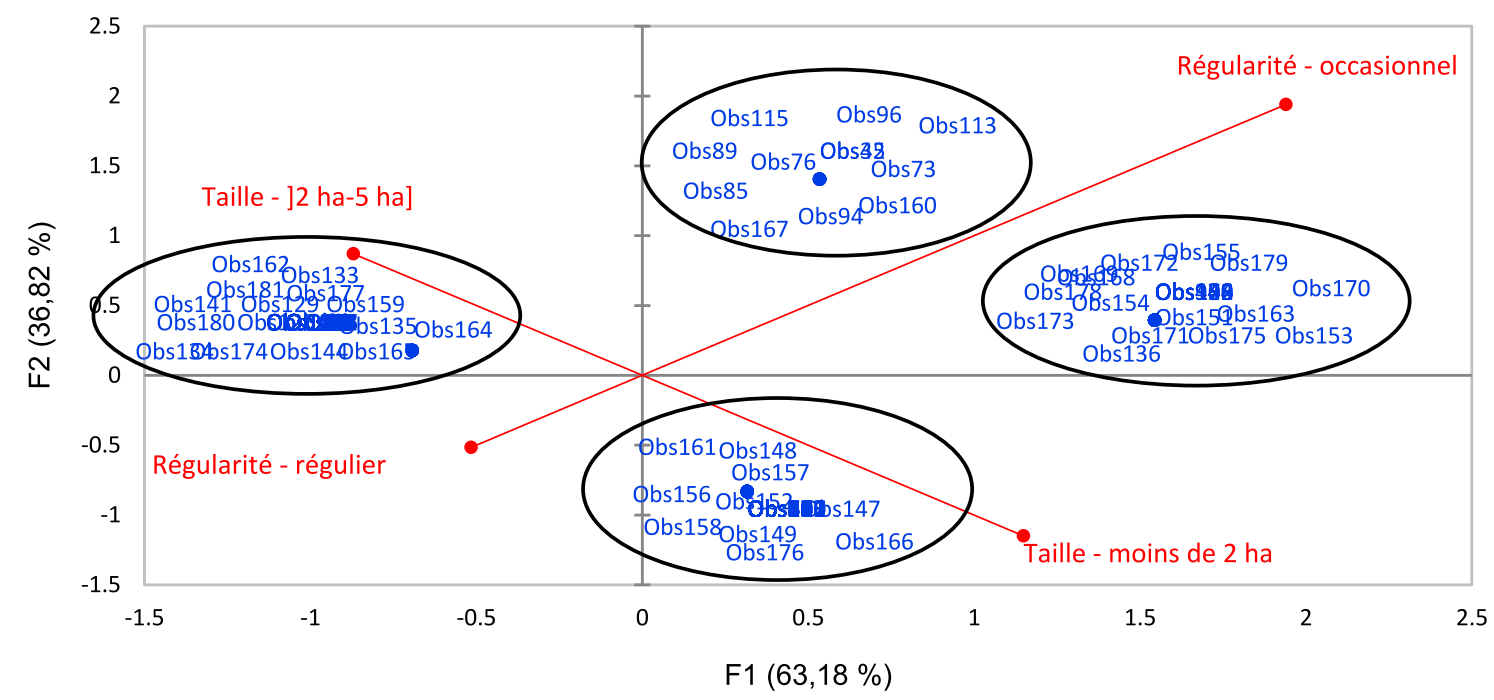

- Variables actives

- Observations actives

Fig. 3. Axes 1 et 2 de l'ACM présentant les relations entre la superficie et la régularité de la production de TI.

Fig. 3. Axes 1 and 2 of the ACM presenting the relationships between area and CT production regularity.

les agriculteurs qui le demandent. Cette contrainte constitue la principale raison pour laquelle les agriculteurs, notamment les plus petits, vendent une partie de leur production sur le marché au comptant, pour financer les frais de récolte. Les délais d'attente aux points de livraison de la CAB sont courts $(30 \mathrm{~h})$, comparés à ceux des concurrents installés à Guelma $(60 \mathrm{~h})$, ce qui permet de réduire les pertes post-récole.

Comme la $\mathrm{CAB}$, les agriculteurs partagent tous le même intérêt pour la subvention, principale motivation de leur adhésion au dispositif contractuel - plus de $70 \%$ des contractants interrogés déclarent avoir signé un contrat pour accéder à la prime. Par contre, les exploitants ne partagent pas tous le même intérêt pour les services proposés par la CAB dans les contrats de production (Tab. 3). Leur participation à l'agriculture contractuelle et leur choix du type de contrat dépendent de leurs objectifs propres, de leur dotation en ressources et de leur éligibilité aux contrats de production.

Les objectifs des petits agriculteurs, qui déterminent leurs besoins pour la production et la commercialisation, diffèrent selon le degré de spécialisation dans la tomate industrielle et les superficies cultivées. Comme le montrent les résultats de l'ACM (Fig. 3), ces différences structurelles et fonctionnelles permettent de distinguer quatre catégories de petits producteurs : les petits ([2-5 ha]) et les très petits $(\leq 2 \mathrm{ha})$ producteurs spécialisés dans la tomate industrielle, les petits ([2-5 ha]) et les très petits $(\leq 2 \mathrm{ha})$ producteurs occasionnels de tomate industrielle (cultivant la tomate industrielle moins de trois campagnes entre 2009 et 2015).

Les agriculteurs cultivant régulièrement la tomate industrielle sont relativement plus intéressés par l'amélioration de leur itinéraire technique. En cas de besoin, les agriculteurs exploitant des superficies réduites (moins de 2 ha selon les normes locales) préfèrent recourir aux prêts entre particuliers, pour mobiliser de petites sommes à moindres coûts de transaction. Ceux exploitant des superficies relativement plus importantes ([2-5 ha]) souhaitent généralement accéder aux
CPA-A. Les producteurs occasionnels ne sont pas dans une logique de pérennisation et de développement de la culture et ne sont donc pas intéressés par les contrats de production nécessitant une mise en culture continue de la tomate industrielle. Ils sont plus motivés par un accès rapide au revenu de la vente. Ceux exploitant des superficies réduites préfèrent rester hors contrat, car les petites quantités produites sont faciles à écouler sous le nom d'un autre contractant. Ils accèdent ainsi à la prime sans engager un coût de contractualisation. Ceux exploitant des superficies relativement plus importantes sont contraints de s'engager dans le contrat commercial afin de réduire les risques de commercialisation (Fig. 4).

$\mathrm{Du}$ fait de leur poids dans la structure agraire locale, les petits producteurs sont toujours majoritaires dans le contrat de commercialisation (Fig. 5). Les petits producteurs restés hors contrat de commercialisation en 2014 sont relativement peu nombreux (115/700). Plus de $73 \%$ d'entre eux n'ont jamais participé formellement au dispositif contractuel. Près de la moitié de ces producteurs hors contrat cultivant de petites superficies (moins de $2 \mathrm{ha}$ ) ont vendu leur récolte à travers un proche qui a signé un contrat. Seulement $15 \%$ sont restés hors contrat par préférence pour le marché au comptant, principalement du fait du paiement immédiat. D'autres, qui exploitent des parcelles en faire-valoir indirect, n'ont pas adhéré au contrat de commercialisation par le passé faute de contrat de location écrit, exigé avant 2014.

La part des petits producteurs dans le CPA-A est relativement réduite (Fig. 6). Certains des petits producteurs (42) ont demandé à accéder à ce contrat, mais ont vu leur demande rejetée par la $\mathrm{CAB}$ (Fig. 4) et accusent parfois l'entreprise de pratiquer une agriculture contractuelle à deux vitesses, défavorable aux petits producteurs. Cette hypothèse est pourtant affaiblie par quelques constats :

- refus se référant toujours aux conditions d'éligibilité;

- participation d'un grand nombre de petits agriculteurs au CPA-A ; 


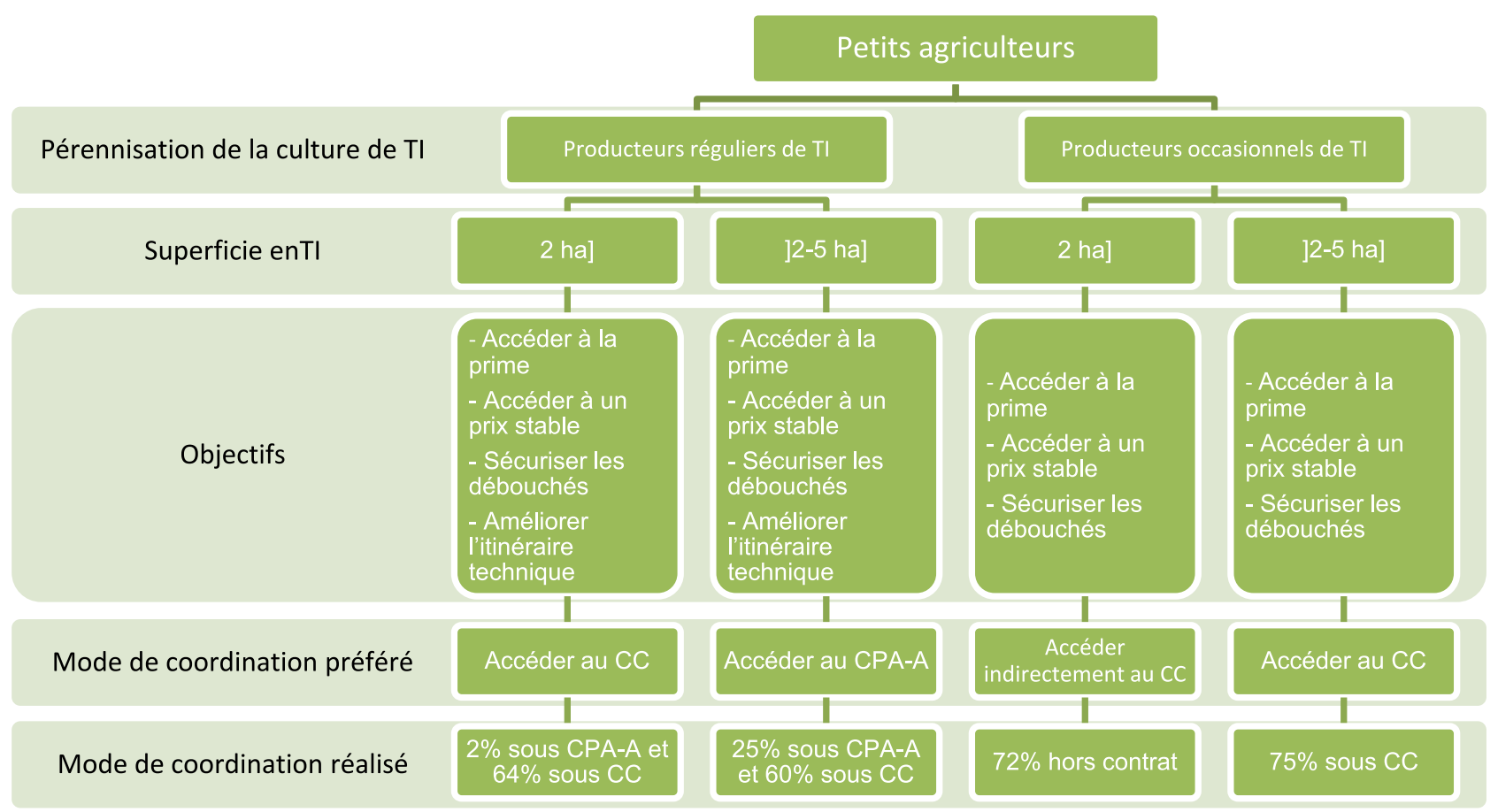

Fig. 4. Objectifs et choix des petits producteurs vis-à-vis de l'agriculture contractuelle.

Fig. 4. Objectives and choices of smallholders in relation to contract farming.

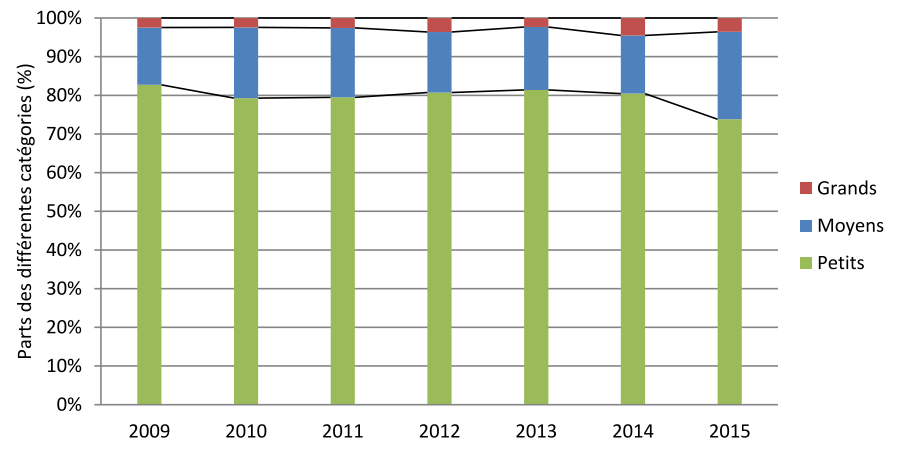

Fig. 5. Évolution des différentes classes de superficie des adhérents au contrat commercial.

Fig. 5. Evolution of the different surface area classes of marketing contract members.

- augmentation du nombre des petits producteurs dans le CPA-A ; avec le temps, les exploitants récemment engagés dans la culture de la tomate industrielle, souvent avec de petites superficies, arrivent à justifier l'ancienneté de quatre ans exigée par la $\mathrm{CAB}$;

- faible engouement des petits producteurs, notamment les très petits, pour les services de la CAB. En 2014, $37 \%$ des producteurs éligibles au CPA-A sont restés volontairement sous contrat de commercialisation. Certains (10) avaient opté pour le CPA-A pour une campagne lors de laquelle ils avaient des difficultés financières, puis sont revenus au contrat de commercialisation. Quelques-uns (3) estiment que les services proposés par l'entreprise (notamment les plants en motte) sont «de mauvaise qualité» ou « surfacturés ».

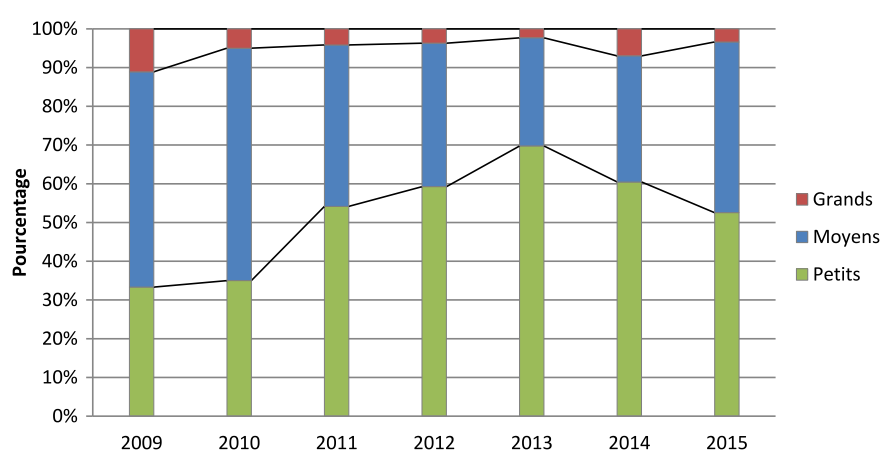

Fig. 6. Évolution des différentes classes de superficie des adhérents au CPA-A.

Fig. 6. Evolution of the different surface area classes of CPA-A members.

\section{Conclusion}

À la différence de ce que documente généralement la littérature sur l'agriculture contractuelle, la participation massive au contrat de commercialisation proposé par l'État est principalement le résultat de la mise en place des subventions. Ces subventions réduisent le coût de fonctionnement du contrat de commercialisation en constituant un garant pour le respect des engagements des agriculteurs et rendent la coordination via le contrat de commercialisation avantageuse pour les deux acteurs, notamment en termes de prix et de risque. Il convient de noter ici que cette orientation de la $\mathrm{CAB}$ vers la production locale plutôt que l'importation du triple concentré s'est trouvée en partie renforcée lors de la pandémie de 2020, qui a fortement perturbé les marchés 
internationaux (en termes de prix, de transport et de disponibilité du produit).

La CAB, pour renforcer son approvisionnement local en tomate industrielle (qui permet une production à plus faible coût et de meilleure qualité), propose, parallèlement au contrat de commercialisation, des contrats de production. Il s'agit pour elle d'attirer et de fidéliser les agriculteurs de la région, y compris les petits, et de les aider à augmenter leur niveau de production via l'amélioration des rendements et l'extension de leurs superficies. Une faible participation des petits producteurs à ces contrats a cependant été constatée. Le décryptage de leurs choix montre que cela vient généralement des objectifs propres de ces agriculteurs, qui font souvent preuve d'un faible engouement pour les contrats de production dans la mesure où ils parviennent généralement à financer sans difficulté majeure les superficies réduites qu'ils exploitent. De son côté, la $\mathrm{CAB}$ ne dresse aucune barrière structurelle, de superficie notamment, pour l'accès au contrat de production. Là aussi, la prime, payée ex post, et qui constitue donc une garantie forte contre l'opportunisme (relativement à la livraison de la production et au remboursement des crédits), permet à l'entreprise de maîtriser les coûts de transaction et de rendre plus accessibles ses contrats de production.

$\mathrm{Au}$ vu du rôle central joué par la prime dans les pratiques contractuelles, on peut s'interroger sur l'avenir de l'agriculture contractuelle en Algérie si l'État se retire, ainsi que sur la place des petits producteurs dans les dispositifs contractuels s'ils étaient maintenus malgré la suppression de la prime, et sur la capacité des entreprises à passer à une deuxième génération de contrats de production gagnant-gagnant. En l'état actuel des choses, d'autres questions font sens, comme les effets de l'agriculture contractuelle sur la coordination des transactions entre les acteurs de la filière ou sur les performances productives et économiques des agriculteurs. Le champ d'investigation sur la question de l'agriculture contractuelle dans la filière de la tomate industrielle en Algérie reste donc largement ouvert.

\section{Références}

Assassi S, Daoudi A, Lejars C. 2017. Les profits «excessifs» des commerçants de fruits et légumes en Algérie: réalité ou préjugé infondé? Le cas de la tomate primeur à Biskra. Cahiers Agricultures 26(2): 25002. DOI: 10.1051/cagri/2017009.

Baci L. 1999. Les réformes agraires en Algérie. Options Méditerranéennes 36: 285-294.
Barrett C, Bachke M, Bellemare M, Michelson H, Narayanan S, Walker T. 2012. Smallholder participation in contract farming: comparative evidence from five countries. World Development 40: 715-730. DOI: 10.1016/j.worlddev.2011.09.006.

Bellemare M, Bloem J. 2018. Does contract farming improve welfare? A review. World Development 112: 259-271. DOI: 10.1016/j.worlddev.2018.08.018.

Burnod P, Colin J. 2012. Grands investissements agricoles et inclusion des petits producteurs : leçons d'expériences dans 7 pays du sud. Land Tenure Working Paper 23. Rome/Montpellier : FAOCIRAD.

Ito J, Bao Z, Su Q. 2012. Distributional effects of agricultural cooperatives in China: exclusion of smallholders and potential gains on participation. Food Policy 37: 700-709. DOI: 10.1016/j. foodpol.2012.07.009.

Le Roux B, Rouanet H. 2010. Multiple correspondence analysis. Thousand Oaks, CA: SAGE Publications, Inc. DOI: 10.4135/ 9781412993906.

Minot N, Sawyer B. 2016. Contract farming in developing countries: theory and practice, and policy implications. In: Devaux A, Torero $\mathrm{M}$, Donovan J, Horton D, eds. Innovation for inclusive value chain development: successes and challenges. Washington DC (USA): IFPRI, pp. 127-158.

Moustier P. 2012. Organisation et performance des filières alimentaires dans les pays du sud: le rôle de la proximité. Synthèse des travaux pour l'habilitation à diriger des recherches. Montpellier, France: CIRAD. https://agritrop.cirad.fr/563989/1/ document_563989.pdf.

Prowse M. 2012 . Contract farming in developing countries: A review. À Savoir 12. Paris, France: Agence française de développement (AFD).

Reardon T, Barrett C, Berdegue J, Swinnen J. 2009. Agrifood industry transformation and small farmers in developing countries. World Development 37(11): 1717-1727. DOI: 10.1016/j.world dev.2008.08.023.

Recensement général agricole (RGA). 2003. Rapport général des résultats définitifs. Alger, Algérie : Ministère de l'Agriculture et du Développement rural.

Roy E. 1963. Contract farming. USA, Danville, IL: The Interstate Printers and Publishers.

Soullier G, Moustier P. 2018. Impacts of contract farming in domestic grain chains on farmer income and food insecurity: contrasted evidence from Senegal. Food Policy 79(C): 179-198. DOI: 10.1016/j.foodpol.2018.07.004.

Ton G, Vellema W, Desiere S, Weituschat S, D'Haese M. 2018. Contract farming for improving smallholder incomes: what can we learn from effectiveness studies? World Development 104: 46-64. DOI: 10.1016/j.worlddev.2017.11.015.

Citation de l'article : Assassi S, Daoudi A, Colin J-P. 2020. Quelle place pour les petits exploitants dans l'agriculture contractuelle? Le cas de la tomate industrielle en Algérie. Cah. Agric. 29: 26. 